\title{
FROM WEAK TO STRONG COUPLING SUPERCONDUCTIVITY: COLLECTIVE MODES IN A MODEL WITH LOCAL ATTRACTION
}

\author{
T. Kostyrko and R. MiCnas \\ Institute of Physics, A. Mickiewicz University, Grunwaldzka 6, 60-780 Poznań, Poland
}

(Received March 12, 1993)

\begin{abstract}
We discuss the density-density response functions and the collective excitation spectrum for the extended Hubbard model with on-site attraction and arbitrary electron density, in the superconducting ground state. For the short-range intersite interaction we find the sound wave-like modes with the velocity interpolating smoothly between the weak $(v \propto 2 Z t,|U| \ll 2 Z t)$ and strong coupling $\left(v \propto Z t^{2} /|U|,|U| \gg 2 Z t\right)$ limits. The latter agrees with the results obtained from an effective pseudospin Hamiltonian valid in the strong coupling limit. In the weak coupling regime, we obtain that apart from a commensurate charge density wave instability, an increase in the intersite Coulomb repulsion can give rise to a charge density wave incommensurate with the lattice period, away from half-filling. The ground state phase diagram for 2D square lattice, including singlet superconducting ground state, electronic droplet formation, and charge density waves is determined. It is also shown that the energy of collective excitations evolves smoothly from weak to strong coupling limit for a $2 \mathrm{D}$ lattice, in the case of a long-range Coulomb interaction. Finally, the mode coupling effects in the frequency behavior of the density-density response functions are analyzed.
\end{abstract}

PACS numbers: 74.20.-z, 71.28.+d, 73.20.Mf

\section{Introduction}

High-temperature superconductors (HTS) (the cuprates, doped bismuthates and fullerenes) generally exhibit low carrier density, a small value of the Fermi energy $(\propto 0.1-0.3 \mathrm{eV})$, short coherence length $\xi_{0}$ and they are extreme type-II superconductors. The estimates based on the Fermi liquid theory yield $\xi_{0} k_{F} \propto 5-10$ (for 1-2-3 cuprates and fullerenes) which indicates that in contrast to a weak coupling BCS theory, the size of a pair is of order of interparticle distance and that all carriers can be involved in the pairing. Moreover, for many high- $T_{\mathrm{c}}$ materials, regardless of a specific microscopic mechanism leading to pairs, there are several universal trends in the $T_{\mathrm{c}}$ versus condensate density dependence, the $T_{\mathrm{c}}$ dependence of the pressure and the isotope effect coefficient. These features of high- $T_{\mathrm{c}}$ 
materials support the models with short-range, nonretarded attraction (see Ref. [1] for a review and [2]).

One of the central questions regarding the physics of HTS is understanding the evolution from a weak coupling limit of large Cooper pairs to the strong coupling regime of small local pairs with increasing coupling constant and description of intermediate (crossover) regime.

The simplest model of general interest is the extended Hubbard model with on-site attractive interaction $[1,3,4]$, defined by the Hamiltonian

$$
\mathcal{H}-\mu N=\sum_{j m \sigma} t_{j m} c_{j \sigma}^{\dagger} c_{m \sigma}+U \sum_{j} n_{j \uparrow} n_{j \downarrow}+\frac{1}{2} \sum_{j m \sigma \sigma^{\prime}} W_{j m} n_{j \sigma} n_{m \sigma^{\prime}}-\mu \sum_{j \sigma} n_{j \sigma} .(1)
$$

In above: $t_{j m}, W_{j m}$ denote hopping integral and intersite density-density interaction, respectively, between sites $j$ and $m$; $U$ is on-site (attractive, $U<0$ ) Hubbard interaction, $\mu-$ the chemical potential, $N, N_{\mathrm{S}}$ - the number of electrons and lattice sites, respectively. It is a nontrivial model of fermions on the lattice which describes transition from weak coupling BCS-like superconductivity to the strong coupling superconductor, where the superconductivity results from the condensation of hard-core composite charged bosons and is similar to superfluidity of ${ }^{4} \mathrm{IIeII}$ $[1,5]$. Such a model has been considered as an effective model of superconductivity in $\mathrm{CuO}_{2}$ planes $[1,5,6]$, of doped $\mathrm{BaBiO}_{3}[1,7]$ and fullerenes $[8,9]$. For $2 Z t \ll|U|$, i.e. small bandwidth, one has a system of local electron pairs which can undergo superfluid transition to the superconducting state with short coherence length $\xi_{0}(\sim$ size of electron pair function). In the opposite limit of large bandwidth, $2 Z t \gg|U|$, the model exhibits a superconducting state of BCS-type with strongly overlapping electron pairs. In this paper we discuss the density-density response function and collective excitation spectrum of the extended Hubbard model over the superconducting ground state for arbitrary electron concentration [10]. These collective modes are related to fluctuations of electron density and the phase of the superconducting order parameter and they can play a role in thermodynamic and spectral properties of the system. Experimentally, spectroscopies such as electron-energy loss experiments can give a direct measure of these modes. We used the diagrammatic perturbation theory to calculate the density-density response function in the random phase approximation (RPA). The collective excitations at $T=0 \mathrm{~K}$ are analyzed in both weak coupling and strong coupling limits and it is shown that they smoothly interpolate between the limits, for short- and long-range intersite Coulomb interaction. From numerical analysis of the collective modes in 2D square lattice, we determined the ground state phase diagram of the system for nearest-neighbor Coulomb interaction. Finally, the effects of long-range Coulomb interaction and general frequency behavior of the density-density response function are discussed.

\section{Random phase approximation for response functions}

Energies of collective modes related to electron density oscillations are given by the poles of density-density response function [11]:

$$
\tilde{\Pi}(q, t)=-\mathrm{i} \Theta(t) \sum_{\sigma \sigma^{\prime}}\left\langle\left[\rho_{q}^{\sigma}(t), \rho_{-q}^{\sigma^{\prime}}\right]\right\rangle
$$


where $\rho_{q}^{\sigma}$ denotes the Fourier component of an electron density operator $n_{i \sigma}=c_{i \sigma}^{\dagger} c_{i \sigma}$ with spin $\sigma$.

The response functions have been evaluated by means of diagrammatic method in the Nambu formalism [10, 12]. For the time-Fourier transform of the density-density response function one obtains formally exact result

$$
\tilde{\Pi}(q)=\frac{\Pi(q)}{1-\left(U / 2+W_{q}\right) \Pi(q)}
$$

where $q=(q, \omega)$ and $W_{q}$ is the spatial Fourier transform of $W_{i j}$. The polarization part $\Pi(q)$ evaluated in the generalized RPA has the following form [10]:

and

$$
\Pi(q) / 2=A(q)-D(q)+2 U \frac{\Gamma(-q) B(q)+\Gamma(q) B(-q)}{(1-U C(q))(1-U C(-q))-U^{2} D^{2}(q)},
$$

$$
\Gamma(q)=[1-U C(q)] B(-q)+U D(q) B(q) .
$$

The unperturbed susceptibilities $A(q), B(q), C(q)$, and $D(q)$ at $T=0 \mathrm{~K}$ are given by

$$
\begin{aligned}
& A(q, \omega)=\frac{1}{2 N_{\mathrm{S}}} \sum_{k}\left(E_{k-q / 2} E_{k+q / 2}-\lambda_{k-q / 2} \lambda_{k+q / 2}\right) \beta_{k, q}(\omega) \\
& B(q, \omega)=\frac{-\Delta}{4 N_{\mathrm{S}}} \sum_{k}\left(\lambda_{k-q / 2}+\lambda_{k+q / 2}\right) \beta_{k, q}(\omega)-\frac{\omega \Delta}{4 N_{\mathrm{S}}} \sum_{k} \beta_{k, q}(\omega), \\
& C(q, \omega)=\frac{1}{2 N_{\mathrm{S}}} \sum_{k}\left(E_{k-q / 2} E_{k+q / 2}+\lambda_{k-q / 2} \lambda_{k+q / 2}\right) \beta_{k, q}(\omega) \\
& -\frac{\omega}{2 N_{\mathrm{S}}} \sum_{k}\left(\frac{\lambda_{k-q / 2}}{E_{k-q / 2}}+\frac{\lambda_{k+q / 2}}{E_{k+q / 2}}\right) \frac{1}{\left(E_{k-q / 2}+E_{k+q / 2}\right)^{2}-\omega^{2}} \\
& D(q, \omega)=-\frac{\Delta^{2}}{2 N_{\mathrm{S}}} \sum_{k} \beta_{k, q}(\omega)
\end{aligned}
$$

where

$$
\beta_{k, q}(\omega)=\left(\frac{1}{E_{k-q / 2}}+\frac{1}{E_{k+q / 2}}\right) \frac{-1}{\left(E_{k-q / 2}+E_{k+q / 2}\right)^{2}-\omega^{2}}
$$

$E_{k}=\sqrt{\lambda_{k}^{2}+\Delta^{2}}$ is a quasi-particle excitation energy, $\lambda_{k}=\varepsilon_{k}-\bar{\mu}$, and $\varepsilon_{k}$ is the spatial Fourier transform of $t_{i j}$. The gap parameter $\Delta$ and the chemical potential $\mu=\bar{\mu}+U n / 2+W_{0} n\left(n=N / N_{\mathrm{S}}\right)$ are determined from the set of the Hartree-Fock (HFA) self-consistent equations [3]:

$$
\begin{aligned}
& 1=-\frac{U}{2 N_{\mathrm{S}}} \sum_{k} \frac{1}{E_{k}}, \\
& 1-n=\frac{1}{N_{\mathrm{S}}} \sum_{k} \frac{\lambda_{k}}{E_{k}} .
\end{aligned}
$$


The polarization part $I(q, \omega)$ is a real quantity provided that $\omega<\min _{k}\left(E_{k}+\right.$ $E_{k+q}$ ) where the last quantity determines a boundary of a quasi-particle continuum. Below this boundary and for small $q, \omega$, we obtain

$$
\Pi(q, \omega)=q^{2} \frac{L(q / q)}{\omega^{2} M_{1}-q^{2} M_{2}(q / q)} .
$$

In above: $L(q / q), M_{1}, M_{2}(q / q)$ are lattice sums depending on the one-electron dispersion $\varepsilon_{k}$ only, not on $W_{q}$, and they are given by

$$
\begin{aligned}
& L(q / q)=\frac{\Delta^{2}}{U} S_{2}(\hat{\boldsymbol{q}})\left(\Delta^{2} S_{0}^{2}+U^{2} S_{1}^{2}\right), \\
& M_{1}=\Delta^{2} S_{0}^{2}+U^{2} S_{1}^{2}, \quad M_{2}(q / q)=\Delta^{2} U^{2} S_{2}(\hat{q}) S_{0},
\end{aligned}
$$

where $q=\hat{q}|q|$ and

$$
S_{0}=\frac{U^{3}}{N_{\mathrm{S}}} \sum_{k} \frac{1}{E_{l}^{3}}, \quad S_{1}=\frac{U^{2}}{N_{\mathrm{S}}} \sum_{k} \frac{\lambda_{k}}{E_{k}^{3}}, \quad S_{2}(\hat{q})=\frac{U}{N_{\mathrm{S}}} \sum_{k} \frac{\left(\hat{q} \cdot \nabla \varepsilon_{k}\right)^{2}}{E_{k}^{3}} .
$$

\section{Collective modes in the system with short-range intersite interaction}

\subsection{Evolution of collective excitalions spectrum}

In the case of long-wave excitations and a short-range interaction $\left(W_{0}<\infty\right)$ the collective modes make a gapless branch $\Omega_{q}^{2}=v^{2} q^{2}$ with velocity $v$ given by

$$
v^{2}=\Delta^{2} S_{2}(\hat{q}) \frac{U^{2} S_{0}+\frac{1}{2}\left(1+2 W_{0} / U\right)\left(\Delta^{2} S_{0}^{2}+U^{2} S_{1}^{2}\right)}{\Delta^{2} S_{0}^{2}+U^{2} S_{1}^{2}}
$$

The existence of this acoustic branch follows from finite range of interaction in the system and continuous degeneracy of the superconducting ground state and exemplifies a nonrelativistic Goldstone theorem [14]. For a quadratic dispersion of the one-electron spectrum, Eq. (15) leads in the weak- $U$ limit to the result closely resembling a weak-coupling formula of Anderson for BCS superconductor [15]:

$$
v^{2}=\frac{1}{d} v_{\mathrm{F}}^{2}\left[1+\left(U+2 W_{0}\right) \mathcal{N}\left(\varepsilon_{\mathrm{F}}\right)\right],
$$

where $d$ is the lattice dimension and $\mathcal{N}\left(\varepsilon_{\mathrm{F}}\right)$ is the density of states per spin at the Fermi level. In general, the velocity of the collective modes as a function of $U$, goes smoothly from the value of order of the bandwidth, for $|2 Z t / U|>1$, to the result of the strong coupling theory (the Bogolyubov mode) [1] which is based on an effective IIeisenberg IIamiltonian exact (for $|t / U| \ll 1)$ up to terms of order $\iota^{2} /|U|:$

$$
v^{2}=\frac{8 Z t^{4}}{U^{2}}\left(1+\frac{W|U|}{2 t^{2}}\right) n(2-n)
$$

( $W$ is the nearest neighbor (NN) interaction). This conclusion has been reached analytically in our previous work [13] (see also [10, 16-18]) to hold for all alternating 
lattices. The fact that RPA interpolates smoothly between the two limits is related to an analogous behavior of the IIartree-Fock (BCS) ground state $[3,19,10]$ above which the collective modes are created in the present theory. Thus, we conclude that there is no sharp distinction between the weak- and strong coupling superconductivity in the negative- $U$ Hubbard model at $T=0 \mathrm{~K}$.

The overall $q$-dependence of $\Omega_{q}$ depends on electron band filling and it has been discussed in detail by us in Ref. [10] (see also [18]). For $n=1$ and $W_{q}=0$, $\Omega_{q}$ plot displays the complete softening of the collective excitation branch for $q \rightarrow M=(\pi, \pi)$ for arbitrary $U$. This reflects the degeneracy of the superconducting $s$-wave state and a commensurate charge density wave (CDW) state which is the exact property of the simple Hubbard Hamiltonian for the half-filled band. As $n$ starts to depart from unity, the absolute minimum at $q=M$ turns to a local one, then at some critical electron density $(n=1-\sqrt{3} / 3$ in the strong $|U|$ limit) it changes to a local maximum. The exact energy of the collective mode for this particular wave vector is given by $\Omega_{q}=U-2 \mu$, as a result of the pseudospin symmetry of the Hubbard Hamiltonian [10].

The typical behavior of $\Omega_{q}$ along the $\Gamma M$ direction of the Brillouin zone (BZ) for the 2D tight-binding square (SQ) lattice and for $n=0.4$ is shown in Fig. 1. The values of $U$ correspond to: the weak coupling $(U=-2.4|t|$, Fig. 1a), intermediate $(U=-3|t|$, Fig. 1b) and intermediate to strong coupling $(U=-4|\ell|$ and $U=-8|t|$, Fig. 1c) ranges. A linear $q$-dependence of $\Omega_{q}$ is clearly seen in the small- $q$ range. In the case of strong attraction the dispersion curves approach values obtained with a help of the effective pseudospin Hamiltonian $[3,10]$, which are given by

$$
\Omega_{q}=\frac{2 Z t^{2}}{|U|} \sqrt{\left(1-\gamma_{q}\right)\left[1-\gamma_{q}\left(2 n^{2}-4 n+1\right)\right]}, \quad \gamma_{q}=\frac{1}{Z} \sum_{\delta} \mathrm{e}^{\mathrm{i} q \cdot \delta}
$$

( $\delta$ - an elementary unit lattice vector). A maximum value of the collective excitation energy is then of order $v \propto t^{2} /|U|$ which is far below the boundary of the quasi-particle excitations continuum, $\min _{k}\left(E_{k}+E_{k+q}\right)>2 \Delta \approx|U|$. The picture changes in the weak-coupling limit. The boundary of quasi-particle continuum decreases whereas maximum value of $\Omega_{q}$ increases with decrease in $|U|$ and finally the dispersion curve of $\Omega_{q}$ sticks to the continuum.

Figure 1 shows the evolution of the excitation spectrum with increasing $U$ in the absence of intersite Coulomb interaction. For weak coupling there exists some critical value of $q, q_{\mathrm{c}} \approx \pi \Delta / \hbar v_{\mathrm{F}}$, for which the collective mode reaches the one-particle continuum and for $q>q_{c}$ but close to the zone boundary there is a splitting of the mode (Fig. 1a). In this case the pair breaking excitations will dominate thermodynamics. With increasing $|U|$, in the crossover regime, there is a qualitative change in the spectrum and the collective mode splits from the continuum for all $q$ (Fig. 1b). In this crossover regime we expect mode coupling effects and low-temperature thermodynamics will be determined by both pair breaking excitations and collective modes. Finally, for large $|U|$, the collective mode approaches the strong coupling theory result of Eq. (18) in the pair Bose condensation regime, and it will drive superfluid transition (Fig. 1c). The approximate boundary between the BCS and pair Bose condensation regimes can be located within the 


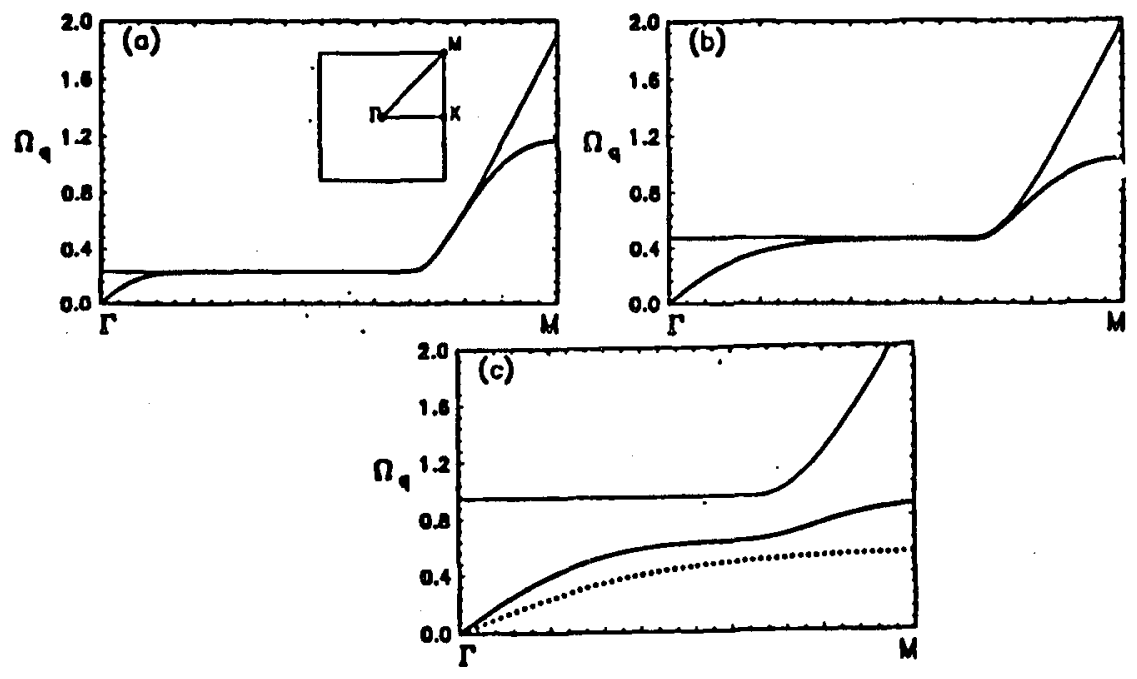

Fig. 1. The energy of the collective modes $\left(\Omega_{q}\right)$ as a function of the wave vector $(q)$ along the direction $\Gamma M$ for the 2D square lattice for $W=0, n=0.4$ and four values of $U$. (a) $U=-1.2$, (b) $U=-1.5$, (c) $U=-2$ and $U=-4$ (dotted line). The inset in Fig. 1a shows BZ for the 2D square lattice. The boundary of the one-particle excitations continuum is shown as a thin solid line. Here and in the next figures we use $2|t|$ as energy unit, unless specified.

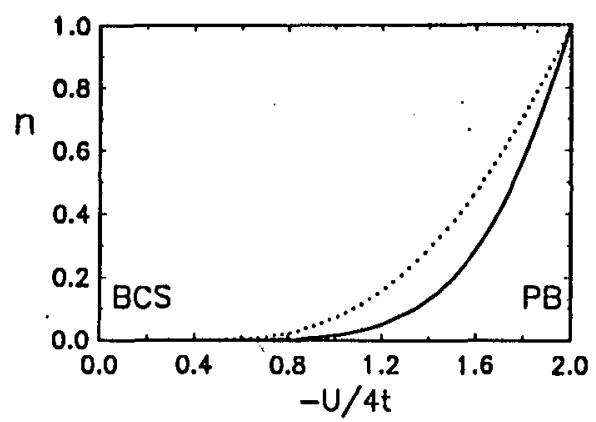

Fig. 2. A line separating BCS and pair Bose condensation (PB) regime from the HFA for $2 \mathrm{D}$ square lattice. The dotted line is for the square density of states.

HFA from the requirement that the chemical potential reaches the bottom of the band, and it is shown in Fig. 2.

\subsection{Instability with respect to $C D W$}

For a critical value of $W_{q}$ depending upon the $|U / t|$ ratio and the electron 
density $n$, the collective mode energy can soften completely for some critical wave vector $q^{\mathrm{c}}$. This effect is the most clearly seen in the strong- $U$ limit where $\Omega_{M}$ goes to zero first for

$$
W_{0}^{c}=\frac{2 Z t^{2}}{|U|} \frac{(1-n)^{2}}{1-(1-n)^{2}}
$$

Softening at $q^{c}=M$ means that the simple $s$-wave state becomes unstable with respect to formation of the commensurate CDW. In a resulting ground state electron charge density alternates along lattice directions, and the superconducting order parameter is still non-zero [3]. The situation is however different in the small- $U$ limit where the critical value of the wave vector depends almost linearly on electron density. From Eq. (3) the $W_{0}^{c}$ can be determined as an absolute minimum in the $\mathrm{BZ}$ of a function

$$
W_{\boldsymbol{q}}^{c}=\frac{1}{\gamma_{\boldsymbol{q}}}\left(\frac{1}{\Pi(\boldsymbol{q}, 0)}-\frac{U}{2}\right)
$$

We thus find an instability of singlet superconducting state toward a new superconducting state accompanied by the incommensurate CDW for $W_{0}>W_{0}^{c}$. Appearance of the incommensurate CDW is a novel feature of a phase diagram of the extended Hubbard model which has not been previously discussed.

For the negative $W_{0}$ the system is unstable toward a phase separation or electron "droplet" formation. With increasing $\left|W_{0}\right|$ the velocity $v$ decreases and upon reaching a critical value of $W_{0}=W_{0}^{d}$ it becomes imaginary for $\left|W_{0}\right|>\left|W_{0}^{d}\right|$ indicating instability of the superconducting state.

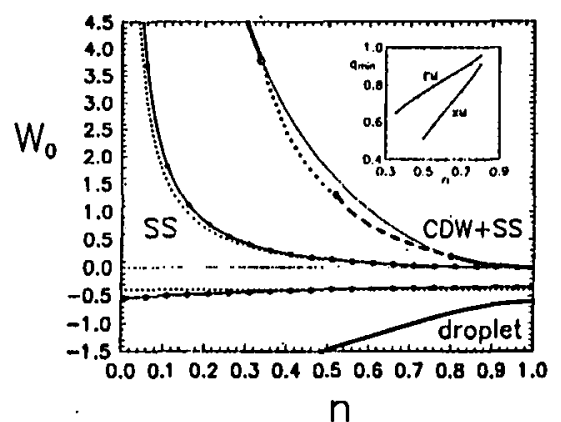

Fig. 3. Ground state phase diagram of the extended Hubbard model with $U<0$ in the $W_{0}-n$ parameters plane for two values of $U(U=-1$ : solid lines; $U=-5$ : empty circles and also tiny dashed line showing the limit of the strong-coupling theory). The $s$-wave superconducting (SS) state is stable between a line of "droplet" instability (for $W_{0}<0$ ) and a line of CDW instability (for $W_{0}>0$ ) for each $U$. Softening of $\Omega_{q}$ along the $X M(\Gamma M)$ direction of the $\mathrm{BZ}$ takes place along the heavy dashed (heavy dotted) curve. In the rest of these curves $\Omega_{q}$ softens exactly at the corner of the BZ first for $W>0$. On the thin solid line $\Omega_{M}$ goes to zero for $U=-1$. The inset shows the minimal values of $q$ (in units of $\pi$ ) versus $n$, for which the $\Omega_{q}$ softens along the $X M$ and $\Gamma M$, directions of the $\mathrm{BZ}$. 
Our RPA analysis of the energy of $\Omega_{q}$ allows us to determine boundaries separating the simple $s$-wave superconducting phase and the other discussed phases in the whole range of $n$ and $|t / U|$. The resulting phase diagram for the NNSQ lattice with $W_{q}=\frac{1}{2} W_{0}\left[\cos \left(k_{x}\right)+\cos \left(k_{y}\right)\right]$ is shown in Fig. 3 . As one can see, the present results are very close to those obtained within the strong coupling theory already for $|U / 2 Z t|=1.25$. In the weak-coupling limit the phase diagram is qualitatively improved by including incommensurate CDWs as compared to that given by the simple Hartree-Fock theory [3].

\section{Effects of long-range Coulomb interactions}

It is well known that in the long-wave limit the long-range Coulomb interactions push the collective modes up to a region of ordinary plasma oscillations (Anderson [15]). This result is valid for isotropic 3D systems and it can easily be seen from our general expressions for the response function Eqs. (3)-(9). The $1 / q^{2}$ divergence of the Fourier transform of the intersite Coulomb term $W_{q}=4 \pi e^{2} / q^{2}$, is canceled by $q^{2}$ term in the numerator of the polarization part (Eq. (12)) leading to a constant term in the denominator of the response function (Eq. (3)) in the limit $|q| \rightarrow 0$. As a consequence the response function can have a divergence for a finite value of $\omega$ only.

In the strictly 2D system the Fourier transform of the long-range term changes to $W_{q}=2 \pi e^{2} /|q|$ which no longer cancels $q^{2}$ term in the numerator of Eq. (12). As a result, one has an "acoustic" plasmon branch with a square-root dispersion

$$
\Omega_{q}=\sqrt{|q| 2 \pi e^{2} \Delta^{2} S_{2} / U}
$$

For the quadratic one-electron dispersion and $\Delta \rightarrow 0$ our calculations reproduce familiar result for $2 \mathrm{D}$ interacting electron gas

$$
\Omega_{\boldsymbol{q}}=\sqrt{2 \pi n e^{2}|q| / m^{*}}
$$

(Crandall [20]). In the opposite limit of the strong on-site attraction we again obtain the result of the strong coupling theory [1]:

$$
\Omega_{q}=\sqrt{2 \pi e^{2}|q| 4 t^{2} n(2-n) /|U|} .
$$

This extends our conclusion about a continuous transition between weak- and strong- $U$ limit to the case of the long-range intersite interactions. Finally, in the particular case of NNSQ lattice we derive a simple explicit formula for $\Omega_{q}$ which interpolates between the two limits

$$
\Omega_{q}=\left(|q| \pi e^{2} \int \mathrm{d} \varepsilon \mathcal{N}(\varepsilon) \frac{\varepsilon(\varepsilon-\bar{\mu})}{\sqrt{(\varepsilon-\bar{\mu})^{2}+\Delta^{2}}}\right)^{1 / 2}
$$

\subsection{D latlice and $3 D$ Coulomb inleraction}

Let us consider now a system of $2 \mathrm{D}$ layers with the isotropic long-range Coulomb interaction within the layers and between them - which is roughly the case of the copper-oxide superconductors. If the interlayer hopping is negligibly 
small, the polarization function does not depend on $q_{\perp}$ (the component of $q$ perpendicular to the plane) and takes the following form:

$$
\Pi(q, \omega)=q_{\|}^{2} \frac{L(q / q)}{\omega^{2} M_{1}-q_{\|}^{2} M_{2}(q / q)}
$$

The Coulomb potential can be approximately represented by $W_{q}=4 \pi e^{2} /\left(q_{\|}^{2}+q_{\perp}^{2}\right)$. In such a case, for $q_{\|} \ll q_{\perp}$, the density-density response function may have a pole inside the superconducting gap, and

$$
\omega_{q}=q_{\|} \sqrt{\frac{U L / 2+M_{2}+4 \pi e^{2} L / q_{\perp}^{2}}{M_{1}}},
$$

where $L=L(0), M_{2}=M_{2}(0)$. This conclusion is in agreement with Fertig and Das Sarma [21]. The interplanar hopping will try to move the collective mode out of the gap. There will be a critical value of interplane-to-intraplane hopping ratio for which the density oscillations with finite $q_{\perp}$ and $q_{\|} \rightarrow 0$ would be pushed out from the gap. This is a highly interesting case involving strong competition of pair breaking excitations and collective modes with relevance for superconducting superlattices.

\section{Frequency dependence of the density response function}

In this section we report our recent numerical results concerning the full frequency dependence of the density-density response functions in the presence of intersite Coulomb interaction at $T=0 \mathrm{~K}$ for the case of $2 \mathrm{D}$ lattice with tight binding spectrum.

There are three different domains of the frequency dependence of $\tilde{\Pi}(q, \omega)$ :

i) For $\omega<\min _{k}\left(E_{k}+E_{k+q}\right)$, i.e. below the one-particle continuum boundary, all components of the zero-order response function (Eqs. (6)-(9)) are real functions of the frequency and so is the density response function (given by Eqs. (3),(4)). At the singularity point $\operatorname{Im} \tilde{\Pi}(q, \omega)$ exhibits the $\delta$-like divergence corresponding to the appearance of the collective mode with given wave vector $q$, which describes coherent phase and electron density oscillations. In this frequency range the collective mode can propagate without damping for $T=0 \mathrm{~K}$.

ii) For $\min _{k}\left(E_{k}+E_{k+q}\right)<\omega<\max _{k}\left(E_{k}+E_{k+q}\right)$, the zero-order response functions possess non-vanishing imaginary contributions, which can be comparable to the real ones. The collective modes can no longer propagate without damping and dissolve into pairs of one-particle excitations.

iii) For $\omega>\max _{k}\left(E_{k}+E_{k+q}\right)$, i.e. above the one-particle continuum, the collective mode can again propagate without damping, very much like the ordinary plasmon excitation in the normal plase.

In our numerical calculations we treated the frequency as a complex number having small imaginary component, $\mathrm{i} \delta$. Such a procedure allows to avoid problems 

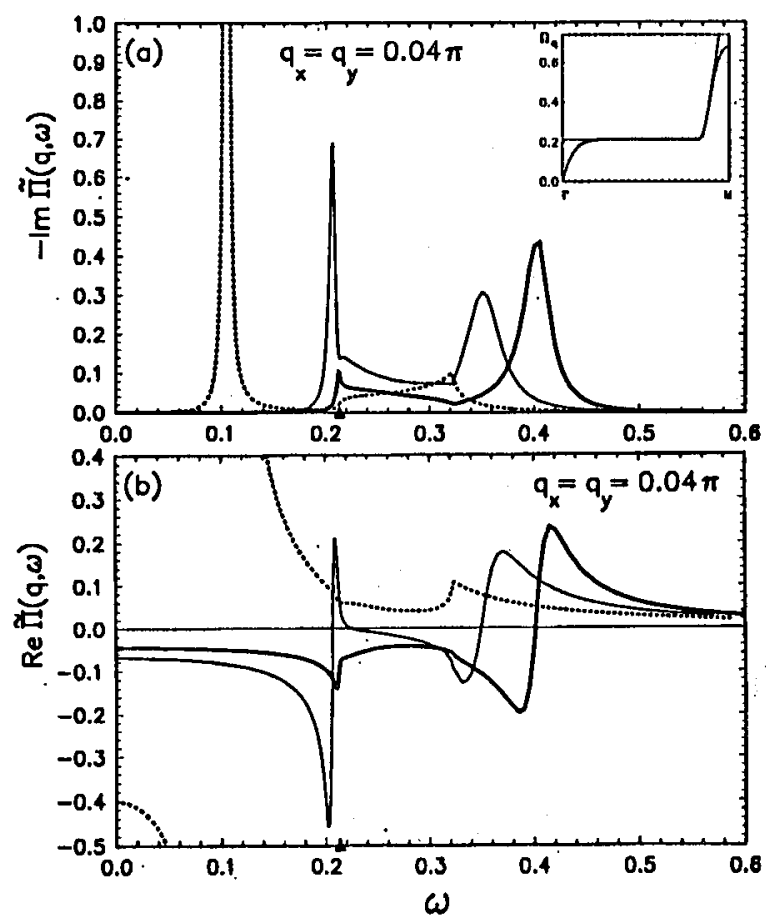

Fig. 4. Behavior of the imaginary (a) and real (b) parts of the density-density response function versus $\omega$ for $2 \mathrm{D}$ square lattice and different values of $3 \mathrm{D}$ intersite Coulomb repulsion. $U=-1, n=0.6$. Dotted line: $W_{q}=0$; thin solid line: $W_{q}=12$; thick solid line: $W_{q}=20$. The position of the one-particle continuum is marked by solid triangle. The inset in Fig. 4a shows the collective mode spectrum for $W_{q}=0$.

with reaching zero in denominators of the expressions for the zero-order response functions for $\omega$ falling inside the one-particle continuum [22]. The numerical values of $\delta$ were adjusted to a limit below which the overall shape of $\omega$-dependence of the response functions did not depend substantially on $\delta$.

The representative results of our computations are presented in Fig. 4. The different values of the Coulomb parameters can correspond to the various values of the component of the wave vector perpendicular to the plane, if a model of 3D Coulomb forces in the system of the interacting layers (following Fertig and Das Sarma [21]) is adopted.

Let us consider first the case of zero intersite Coulomb repulsion. The imaginary part of the full response function remains vanishingly small for $\omega<2 \Delta$ with the exception of the vicinity of $\omega=\omega_{q} \approx 0.1$ where a strong $\delta$-like peak is seen. (The finite width of the peak results here from keeping a small imaginary part of 
$\omega$, as it was explained above.) The real part of $\tilde{\Pi}$ exhibits a $1 /\left(\omega-\omega_{q}\right)$ divergence which is typical of the behavior of the response function in the vicinity of the well-defined collective mode with an infinite lifetime. The peak of $\operatorname{Im} \tilde{I}$ has a considerable spectral weight and apparently dominates over the rest of the spectrum. After crossing the continuum boundary for $\omega=2 \Delta, \operatorname{Im} \tilde{I}$ raises abruptly and then it becomes a monotonuous function of the frequency up to a particular ( $q$-dependent) value of $\omega$ where it displays a van Hove like singularity and next decreases steadily with $\omega[23,21]$. Finally, it vanishes upon crossing a second boundary of the one-particle continuum.

The above picture changes gradually once we turn on the intersite Coulomb repulsion $W$. The increase in $W$ results in a shift of the peak position of the collective mode towards the region of higher energies (see also Eq. (15)). The energy of undamped collective mode ceases to increase upon reaching the vicinity of the one-particle continuum, where the spectral weight of the mode quickly decreases with subsequent increase in the Coulomb repulsion. At the same time the substantial part of the spectral weight of $\operatorname{Im} \tilde{I}$ is moved to the domain of one-particle excitations. Moreover, with further increase in the Coulomb repulsion, a prominent maximum begins to raise well inside the continuum. From observation of the corresponding change of sign of $\operatorname{Re} \tilde{\Pi}$ near the center of this maximum, we can associate this feature with a damped collective mode. In other words, as a result of the interactions between the coherent oscillations of the electron density and the one-particle excitations, we observe a splitting of the spectrum of the collective modes into the undamped part, staying slightly below the continuum boundary and the strongly damped mode formed at some distance above the boundary.

A reason for the discussed behavior of $\operatorname{Im} \tilde{I}$ in the domain of the one-particle continuum can be traced back to a specific frequency dependence of the polarization part $\Pi$. One has, in particular

$$
\operatorname{Im} \tilde{\Pi}(q, \omega)=\frac{\operatorname{Im} \Pi(q, \omega)}{\left[1-V_{q} \operatorname{Re} \Pi(q, \omega)\right]^{2}+\left[V_{\boldsymbol{q}} \operatorname{Im} \Pi(q, \omega)\right]^{2}},
$$

where $V_{\boldsymbol{q}}=U / 2+W_{\boldsymbol{q}}$. For the $\operatorname{Im} \tilde{\Pi}(\boldsymbol{q}, \omega)$ to develop a well-defined peak, not only $\operatorname{Re} \Pi$ should be equal to $1 / V_{\boldsymbol{q}}$, but also $\operatorname{Im} \Pi$ should be significantly less than the real part. In the region between the van Hove singularity and the lower one-particle boundary, $\operatorname{Im} \Pi(q, \omega)$ is comparable to $\operatorname{Re} \Pi(q, \omega)$ and thus vanishing of $\left(1-V_{q} \operatorname{Re} \Pi(q, \omega)\right)$ has not a great impact on the value of denominator of $\tilde{\Pi}(q, \omega)$. After crossing the van Hove singularity point, $\operatorname{Im} \Pi(q, \omega)$ begins to decrease rather quickly and the relatively narrow peak corresponding to a collective mode can appear.

Our conclusions regarding mode coupling effects result from strongly non-monotonous frequency dependence of the polarization function and we expect them to be valid beyond the RPA treatment. It would be of interest to extend the above analysis of charge response functions to the case of superconducting superlattices with anisotropic Coulomb interaction and interlayer couplings.

\section{Acknowledgments}

We would like to thank Prof. S. Robaszkiewicz for very valuable discussions. 
A part of this work has been presented at XVI International School of Theoretical Physics, Ustron 1992, Poland. We gratefully acknowledge the financial support of the Committee for Scientific Research within the projects: 2 P3 02057 04, 200119101 , and 224339102.

\section{References}

[1] R. Micnas, J. Ranninger, S. Robaszkiewicz, Rev. Mod. Phys. 62, 113 (1990).

[2] T. Schneider, H. Keller, Phys. Rev. Letl. 69, 3374 (1992).

[3] S. Robaszkiewicz, R. Micnas, K.A. Chao, Phys. Rev. B 23, 1447 (1981); 24, 1579 (1981); 24, 4018 (1981); 26, 3915 (1982).

[4] R.T. Scalettar, E. Loh, J. Gubernatis, A. Moreo, S. White, D. Scalapino, R. Sugar, E. Dagotto, Phys. Rev. Lett. 62, 1407 (1989).

[5] N.F. Mott, Adv. Phys. 39, 55 (1991); L.J. de Jongh, Physica C 152, 171 (1988); 161, 631 (1989).

[6] A.R. Bishop, P.S. Lomdahl, J.R. Sclirieffer, S.A. Trugman, Phys. Rev. Lell. 61, 2709 (1988).

[7] C.M. Varma, Phys. Rev. Lett. 61, 2713 (1988).

[8] S. Chakravarty, S. Kivelson, Europhys. Lett. 16, 751 (1991).

[9] F.C. Zhang, M. Ogata, T.M. Rice, Phys. Rev. Lett. 67, 3452 (1991); J.A. Wilson, Physica C 182, 1 (1991).

[10] T. Kostyrko, R. Micnas, Phys. Rev. B 46, 11025 (1992).

[11] J.R. Schrieffer, Theory of Superconductivity, Benjamin, New York 1964; A.L. Fetter, J.D. Walecka, Quantum Theory of Many-Particle Systems, McGraw-Hill, New York 1971.

[12] The response functions can also be obtained by the functional differentiation technique of Kadanoff and Baym, see R. Côte and A. Griffin, Preprint, University of Toronto, Toronto 1993.

[13] T. Kostyrko, Phys. Status Solidi B 135, K79 (1986); 143, 149 (1987).

[14] R.V. Lange, Phys. Rev. 146, 301 (1966); J.B. Johansson, J. Phys. C 3, 50 (1970).

[15] P.W. Anderson, Phys. Rev. 112, 1900 (1958); G.P. Angelis, F. Mancini, Nuovo Cimento Lelt. 10, 654 (1974).

[16] K. Nasu, Physica B 143, 229 (1986).

[17] L. Belkhir, M. Randeria, Phys. Rev. B 45, 5087 (1992).

[18] J.O. Sofo, C.A. Balseiro, H.E. Castillo, Phys. Rev. B 45, 9860 (1992).

[19] P. Nozieres, S. Schmitt-Rink, J. Low Temp. Phys. 59 , 195 (1985); M. Randeria, J.-M. Duan, L.-Y. Shieh, Phys. Rev. Lett. 62, 981 (1989); Phys. Rev. B 41, 327 (1990).

[20] R.S. Crandall, Phys. Rev. A 8, 2136 (1973).

[21] H.A. Fertig, S. Das Sarma, Phys. Rev. Lett. 65, 1482 (1990); Phys. Rev. B 44, 4480 (1991).

[22] A similar method was applied by N. Bulut and D. Scalapino, Phys. Rev. B 45, 2371 (1992), in the RPA calculations of the relaxation rate for the $d$ - and $s$-wave superconductor.

[23] R.E. Prange, Phys. Rev. 129, 2495 (1963). 